\title{
Acercamiento a los regímenes internacionales de control de exportaciones: caso aduana mexicana
}

\section{Approach to international export control regimes: Mexican Customs Case}

\author{
Aleida Núñez-García' \\ Universidad Autónoma de Nuevo León \\ aleida.nunezgr@uanl.edu.mx \\ Jorge Isaac Lechuga-Cardozo ${ }^{2^{*}}$ \\ Universidad Sergio Arboleda \\ Jorge.lechuga@usa.edu.co
}

\section{Autor corresponsal.}

Universidad Autónoma de Nuevo León, Facultad de Ciencias Políticas y Relaciones Internacionales, Pedro de Alba S/N. Niños Héroes, Ciudad Universitaria, San Nicolás de los Garza, N.L., MEXICO.

2 Universidad Sergio Arboleda,

Escuela Internacional de Administración y Marketing, Carrera 46 km. 4 vía al mar. Puerto Colombia, COLOMBIA

\section{Resumen}

Propósito: El objetivo de este trabajo es dar a conocer los regímenes internacionales de control de exportaciones aplicables a la aduana mexicana a partir de la teoría del realismo de las relaciones internacionales.

Originalidad/valor: El estudio de los regímenes internacionales de control de exportaciones cobra importancia debido al dinamismo y la necesidad de actualizar las estructuras normativas de seguridad a las políticas aduaneras, particularmente en la aduana mexicana.

Diseño/Metodología: Se realiza un estudio cualitativo aplicando la técnica de revisión de literatura a través del método de análisis bajo un diseño no experimental transversal.

Resultados: La revisión documental permitió enlistar los elementos que integran el marco jurídico internacional del control de mercancías para asegurar y facilitar el comercio.

Implicaciones: Se concluye que es preciso insistir en que México y América Latina se acojan a las políticas internacionales en materia de control de mercancías, para facilitar la seguridad de las mercancías en la cadena de suministro. De esta manera, se contribuye al mejoramiento global en los estándares internacionales de seguridad en el tráfico de bienes.
INFORMACIÓN ARTÍCULO

Recibido: 30 de Octubre 2021

Aceptado: 7 de Enero 2022
Palabras Claves:

Aduanas

Consejo de seguridad

Control de mercancías

Marco de estándares para asegurar y

facilitar el comercio

Regímenes internacionales de seguridad. 


\begin{abstract}
Purpose: The purpose of this paper is to present the international export control regimes applicable to Mexican customs based on the realism theory of international relations.

Originality/value: The study of international export control regimes gains importance due to the dynamism and the need to update security regulatory structures to customs policies, particularly in Mexican customs. Design/methodology: A qualitative study is carried out applying the literature review technique through the method of analysis under a nonexperimental cross-sectional design

Findings: The documentary review made it possible to list the elements that make up the international legal framework for the control of merchandise to ensure and facilitate trade.

Practical implications: It is concluded that it is necessary to insist that Mexico and Latin America adhere to international policies on merchandise control to facilitate the security of merchandise in the supply chain, thus contributing to the global improvement in international standards of security in the traffic of goods.
\end{abstract}

\section{ARTICLE INFO}

Received: 30 October 2021

Accepted: 7 January 2022

\section{Keywords:}

Customs

Customs control

Security Council

Framework of Standards to Secure and

Facilitate Global Trade

International security regimes

\section{INTRODUCCIÓN}

El comercio internacional es una actividad milenaria que se distingue por la importación y exportación de mercancías entre los diferentes países. Para regular la entrada o salida de bienes, los países han establecido entes administrativos denominados aduanas. Las funciones de estas se han modificado producto del dinamismo del comercio internacional. En las últimas dos décadas el tema de la seguridad ha cobrado especial atención en el mundo, y, en particular, en las aduanas. Los países, así como diversos organismos internacionales involucrados en estos temas, han generado políticas y compromisos para salvaguardar el interés nacional.

Por otra parte, las aduanas desempeñan una función social importante debido a que son oficinas públicas en fronteras, litorales y ciudades portuarias que fiscalizan, vigilan y controlan la entrada de mercancías y los vehículos donde estas son transportadas. En México, la legislación, los sistemas, métodos y procedimientos para la regulación del despacho aduanero son competencia de la Administración General de Aduanas (AGA).

En ese sentido, la AGA se define como la autoridad competente para aplicar la legislación que regula el despacho aduanero, como también los sistemas, métodos y procedimientos a los que deben sujetarse las aduanas. El objetivo de este trabajo es dar a conocer los regímenes internacionales de control de exportaciones aplicables a la aduana mexicana, a partir de la teoría del realismo de las relaciones internacionales. Estos enfoques internacionales incluyen las normas de seguridad de la Organización de Naciones Unidas (ONU), de las normas de seguridad de la Organización Mundial de Aduanas (OMA), y los regímenes internacionales de seguridad. Desde el punto de vista de las teorías de las relaciones internacionales, se incluye el realismo. En este contexto, ¿Las políticas aduaneras de México se actualizan respecto de las diversas disposiciones internacionales de seguridad?

El trabajo se divide en cuatro secciones. En primera instancia, se explican conceptos elementales de la teoría de los regímenes internacionales, en particular, los de control de exportaciones y su adaptación en los temas de comercio internacional. Además, se incluyen los fundamentos básicos de la teoría realista con los que se pretende explicar el presente tema. Posteriormente, el texto describe de forma sucinta el funcionamiento del Consejo de Seguridad de la ONU y su aplicación en la operatividad del régimen internacional de seguridad. En tercer lugar, el texto describe el Marco SAFE de la OMA y su aplicación en las aduanas del globo. El texto incluye los alcances para mitigar el problema de seguridad de las mercancías de comercio internacional, particularmen- 
te de nuestro país, por lo que se describe de forma breve la estructura de la aduana mexicana. Además, se incorpora información sobre las sanciones respecto de las mercancías contempladas en los regímenes de control de exportaciones, a través del estudio de los problemas suscitados en otros países. Finalmente, se realizan conclusiones generales a partir de algunas consideraciones.

\section{MÉTODO}

\section{Diseño}

El estudio tiene un enfoque cualitativo, con el propósito de utilizar la recolección y análisis de datos para dar respuesta a la pregunta de investigación. Asimismo, tiene un diseño no experimental, porque las categorías estudiadas se observan de manera natural (Hernández y cols., 2014). Por otro lado, con un alcance interpretativo se intenta encontrar patrones de relación internas en la actualización de las políticas aduaneras de México a los regímenes internacionales de control de exportaciones para llegar a profundizar en el conocimiento de este (Hurtado, 2010). Desde un punto de vista cronológico, el trabajo tiene un diseño transversal, desprovisto de seguimiento en el tiempo y se efectuó en el segundo semestre de 2020 (Hernández y cols., 2014).

\section{Instrumentos}

La elaboración del marco teórico-conceptual de los regímenes internacionales de control de exportaciones caso Aduana de México, consistió en recopilación de veintisiete referencias cuyos argumentos fueron interpretados de forma analítica y crítica.

\section{Procedimiento}

Para comprender el problema de la actualización de las políticas aduaneras de México a los regímenes internacionales de control de exportaciones, se realiza una revisión de literatura del marco jurídico internacional del control aduanero de mercancías a través de fuentes secundarias. En ese sentido, Bernal (2010) señala que este procedimiento permite el análisis de información escrita con el fin de establecer el estado actual de conocimiento del tema, el que depende de la información que se obtiene sin que se altere su sentido. De este modo, se recopila y analiza la información, y se elabora el presente documento a partir del objetivo trazado (Lechuga, Leyva y Núñez, 2019; Lechuga, Núñez y Leyva, 2019).

\section{DESARROLLO DEL TEMA}

\section{Regimenes internacionales}

Es innegable el número de acuerdos internacionales que se suscriben en el mundo. Los sectores, reservas, aranceles, cupos y diversas medidas comerciales de bienes y servicios negociadas quedan a la potestad de los Estados. En ese sentido, Lascurain (2018) señala que las fronteras entre los países son puntos de contacto y cooperación, y la forma de comprender esas interacciones es analizando cómo los gobiernos y sus comunidades trabajan para combatir problemas de diferente naturaleza (por ejemplo: económica, social, política y ambiental). No obstante, las fronteras también son fuentes de tensiones y conflictos.

Derivado de lo anterior y con la finalidad de dar explicación a diversos fenómenos de las relaciones entre países, surge el concepto de regímenes internacionales. Puchala y Hopkins (1983), señalan que el creciente interés del concepto de régimen internacional en la década de 1970 es similar al interés por el concepto de sistema internacional en la década de 1950. En ese sentido, Mesinas (2017) resalta la importancia de los regímenes internacionales porque proveen un marco normativo y regulatorio en las estructuras del Estado.

Por otra parte, el uso del término régimen nos permite señalar y comprender un conjunto de actividades que podrían organizarse de otro modo o entenderse de manera diferente. Por lo tanto, pensar en términos de regímenes también nos alerta sobre los aspectos subjetivos del comportamiento internacional que podrían pasarse por alto en investigaciones más convencionales.

En ese sentido, Krasner (1982) define un régimen internacional como una agrupación de regulaciones y procesos en torno a las relaciones entre países. De igual forma, Puchala y Hopkins (1983) señalan que esta conceptualización es pertinente para la explicación de temáticas diversas dentro de las relaciones internacionales.

Finalmente, existe un régimen en todas las áreas temáticas sustantivas de las relaciones internacionales en las que hay un comportamiento estructurado. Bajo esa línea, si existe regularidad en 
el comportamiento, deben existir algunos tipos de principios, normas o reglas para dar cuenta de ello. Tal comportamiento modelado puede reflejar el dominio de un actor en lugar de un consenso voluntario entre todos los participantes. Por lo tanto, los principios del régimen internacional llegan a coincidir con los valores, objetivos y procedimientos de toma de decisiones del participante 0 participantes preeminentes.

Se han categorizado de diversas formas los regímenes internacionales, por ejemplo, Hasenclever y cols. (2000) los clasifican como: régimen de seguridad, régimen económico, régimen ambiental y régimen de derechos humanos.

\section{Regimenes internacionales de control de exporta- ciones}

Es indiscutible el avance tecnológico de diversos sectores dedicados a la producción de mercancías de uso dual. La preocupación de los Estados respecto de su proliferación implica cambios normativos, cooperación y voluntad de los países, necesarios para ejercer control sobre la producción, venta y distribución de estas mercancías. Equilibrar las regulaciones de tipo no arancelario en las exportaciones de estos bienes debe de considerarse como una prioridad de seguridad nacional. Al respecto, Díaz (2014) señala que el establecimiento de los regímenes de control de exportaciones permitió herramientas multilaterales contra la emergencia de armas de destrucción masiva y vectores de lanzamiento, así como permisos y licencias a ciertas exportaciones de tecnología de doble uso. Estos regímenes armonizan las políticas de control de exportaciones entre los Estados, con relación a las directrices y los bienes sometidos a control a través de unas listas técnicas.

Asimismo, la materialización de los compromisos realizados por México se manifiesta, por una parte, por la publicación de listas técnicas de mercancías sujetas a control, y, por otra, obedecen a directrices internacionales como la resolución de la Asamblea General de la ONU: 64/40 de fecha 12 de enero de 2010 y la resolución 1540 de fecha 5 de noviembre de 2004, emitida por el Consejo de Seguridad de la ONU. México ha contraído obligaciones internacionales como lo refiere la declaración realizada en la página oficial de la Secretaría de Economía:

En este contexto, esta Administración refrenda el compromiso de fortalecer el Sistema Mexicano de Control de Exportaciones mediante la actualización constante del marco normativo nacional en dicha materia, considerando la implementación de las mejores prácticas internacionales aprendidas en colaboración con los miembros de los Regímenes Internacionales de Control de Exportaciones (Secretaría de Economía - SE, s.f.).

Los regímenes internacionales de control de exportaciones se pueden observar en la Tabla 1. Además, contiene los bienes o mercancías controlados, y la membresía de México con cada uno de ellos.

Tabla 1: Regímenes internacionales de control de exportaciones

\begin{tabular}{|c|c|c|c|c|}
\hline Régimen internacional & Bienes controlados & $\begin{array}{l}\text { Estatus } \\
\text { de México }\end{array}$ & $\begin{array}{l}\text { México miembro } \\
\text { desde }\end{array}$ & $\begin{array}{l}\text { No. países } \\
\text { miembros }\end{array}$ \\
\hline Acuerdo de Wassenaar & $\begin{array}{l}\text { Armas Convencionales, Bienes y Tecnologías de } \\
\text { Uso Dual, y tecnologías de uso pacífico y bélico. }\end{array}$ & Admitidos & ene-2012 & 41 \\
\hline $\begin{array}{l}\text { Grupo de Suministra- } \\
\text { dores Nucleares }\end{array}$ & $\begin{array}{l}\text { Bienes y tecnologías nucleares, equipo, material, } \\
\text { software de uso dual y tecnología relacionada. }\end{array}$ & Admitidos & sep-2012 & 46 \\
\hline Grupo de Australia & $\begin{array}{l}\text { Sustancias químicas, agentes biológicos y ele- } \\
\text { mentos y equipos para la fabricación de sustan- } \\
\text { cias químicas y biológicas de uso dual. }\end{array}$ & Admitidos & ago-2013 & 42 \\
\hline $\begin{array}{l}\text { Régimen de Control de } \\
\text { Tecnología de Misiles }\end{array}$ & $\begin{array}{l}\text { Misiles, equipo, software y tecnología relacio- } \\
\text { nada. }\end{array}$ & $\begin{array}{l}\text { Por definir } \\
\text { ingreso }\end{array}$ & pendiente & 34 \\
\hline
\end{tabular}




\section{Aproximación teórica desde las relaciones inter- nacionales: Realismo}

Las teorías que explican las relaciones internacionales poseen premisas y conceptos que ayudan a la comprensión de los fenómenos internacionales. En concreto, la teoría realista explica el sistema internacional desde una visión del poder en el que el Estado como actor principal de las relaciones internacionales maximiza el poder en búsqueda del beneficio propio. Asimismo, el conflicto y la cooperación se dan en un sistema de naturaleza anárquica, es decir, un sistema con ausencia de una autoridad central. En este sentido, la seguridad es uno de los temas trascendentales de la política exterior de los Estados (Person y Rochester, 2000; Schiavon y cols., 2014; Zeraoui y cols., 2014; Velázquez y González, 2016).

Por consiguiente, según los realistas, la seguridad nacional que incluye la gestión del riesgo en el sistema de aduanas es tema de agenda. Por ello, la actualización del marco normativo nacional en temas de seguridad internacional debe ser una prioridad desde el enfoque realista. Con relación a México, se ha contraído el compromiso internacional en la búsqueda de la seguridad en la cadena de suministro al haber un aumento significativo de las empresas certificadas.

\section{Consejo de Seguridad de Naciones Unidas}

Para hacer referencia al Consejo de Seguridad es preciso definir el sistema de Naciones Unidas:

Las Naciones Unidas nacieron oficialmente el 24 de octubre de 1945, después de que la mayoría de los 51 Estados Miembros signatarios del documento fundacional de la Organización, la Carta de la ONU, la ratificaran. La carta se firmó el 26 de junio de 1945 en San Francisco, al terminar la Conferencia de las Naciones Unidas sobre Organización Internacional, y entró en vigor el 24 de octubre del mismo año. En la actualidad, 193 Estados son miembros de las Naciones Unidas, que están representados en el órgano deliberante, la Asamblea Ceneral (ONU, 2020a).

Órganos principales. Para cumplir con sus diversas funciones, la ONU cuenta con una estructura que integra a los principales órganos. De acuerdo con ONU (2020b), estos son: la Asamblea Ceneral, Consejo de Seguridad, Consejo Económico y
Social, Consejo de Administración Fiduciaria, la Corte Internacional de Justicia y la Secretaría de la ONU. Todos ellos se crearon en 1945 al fundarse la ONU.

Consejo de Seguridad. Como se estableció con antelación, el Consejo de Seguridad se creó en el mismo momento de la fundación de la ONU, sin embargo, se inauguró el 17 de enero de 1946 en Londres (ONU, 2020c). El fundamento legal del consejo de seguridad se establece del artículo 23 al 32, del capítulo $V$ de la carta de las naciones. Los artículos 23, 24 y 25 del mismo ordenamiento señalan la composición, funciones/poderes y obligatoriedad, respectivamente (ONU, 2020d).

Objetivos. La Carta de las Naciones Unidas estipula que dentro de las funciones y poderes del Consejo de Seguridad se encuentran mantener la paz y la seguridad, investigar controversias, planificar la dotación de armas, accionar medidas para contrarrestar amenazas a la paz, entre otras (Consejo de Seguridad de las Naciones Unidas, 2020e). Asimismo, con el objetivo de mantener la paz y la seguridad internacional, el Consejo de Seguridad está conformado por 15 miembros de los cuales 5 son permanentes y los 10 restantes removibles por periodo. Los permanentes tienen derecho a voto y veto y los no permanentes solo a voto.

El acato obligatorio de los Miembros sobre las decisiones tomadas en el seno del Consejo de Seguridad implica certeza a las operaciones de comercio internacional. De conformidad con la resolución 1540 del Consejo de Seguridad, y en virtud del Capítulo VII de la Carta de las Naciones Unidas, se establece que todo Estado debe acoger las leyes aplicándolas de manera eficaz y que impida a los ciudadanos cualquier actividad que implique el uso o transformación de armas nucleares, químicas o biológicas, en especial atención a las dedicadas al terrorismo.

De igual forma, determinar si hay una amenaza a la paz mundial o un acto de agresión, y hacer alguna recomendación de parte del Consejo de Seguridad se vuelve el mecanismo Ad Hoc para materializar la seguridad a través de las funciones aduaneras. De esta forma, las aduanas no solo reciben y envían mercancías en los diferentes regímenes aduaneros, sino que contribuyen significativamente a disminuir el riesgo país en el contexto del comercio internacional. 
Marco de estándares para asegurar y facilitar el comercio (SAFE) de la Organización Mundial De Aduanas (OMA)

Origen. Acontecimientos que amenazaron la seguridad mundial como el ataque a las torres gemelas y el Pentágono el 11 de septiembre de 2001 en Estados Unidos, afectaron considerablemente los esquemas de seguridad a nivel mundial. No obstante, fue hasta junio de 2005 en Bruselas, donde la OMA adopta un marco normativo para el SAFE. Este hito dio origen al marco normativo de la seguridad en la gestión de la cadena logística, y el reconocimiento de la lucha contra el terrorismo y el narcotráfico de las Aduanas y las empresas (OMA, 2018).

Importancia/Función. El comportamiento del comercio internacional siempre está en constante cambio. Lo anterior, debido a factores como el desarrollo y/o avance tecnológico y la inseguridad. Respecto a ello, la OMA (2018) señala que al ser el comercio internacional un impulsor de bienestar económico se hace sensible a actos terroristas que amenazan su estabilidad. En este sentido, la administración de aduanas como ente de control y gestión del movimiento internacional de mercancías, debe brindar la seguridad en la cadena logística y facilitar el comercio acorde a los acuerdos vigentes entre países.

\section{Elementos básicos del marco de seguridad}

Dada la posición exclusiva de las aduanas en el globo, estas han ganado terreno en la seguridad con el avance electrónico y manejo de información. La gestión del análisis de riesgo cada vez se afina más para buscar la predictibilidad y la eficacia en las operaciones de comercio exterior. En este contexto, la OMA (2018) en el marco SAFE, instituye cuatro elementos fundamentales, a saber: (1) armoniza información electrónica de manera anticipada de envíos; (2) compromiso en la gestión del riesgo en temas de amenaza a la seguridad; (3) deber de la aduana del país remitente realizar una inspección de la carga y medios de transporte a través de técnicas de detección no intrusivas y (4) las aduanas brindarán beneficios a las empresas que cumplan con normas de seguridad en la cadena logística.

\section{Pilares estructurales del marco de seguridad}

Los sujetos participantes de las operaciones aduaneras, ya sea en el ambiente público o pri- vado, son usuarios directos de la normatividad aduanera. La colaboración entre todos los entes participantes es parte estratégica del objetivo del marco SAFE como lo establece la OMA (2018), a través de sus pilares estructurales relacionados a arreglos entre Aduanas-Aduanas, asociaciones Aduanas-Empresas y Aduanas con otras autoridades. Entre sus múltiples ventajas los pilares facilitan la comprensión y ágil aplicación internacional, asimismo, se encuentran fundamentadas en medidas de seguridad y facilitación de la OMA.

\section{Acuerdos de la red de aduanas}

Es innegable que la colaboración entre las diferentes administraciones aduaneras ha traído resultados positivos. El binomio facilidad versus control, es posible sostenerlo a través de información fidedigna entre las aduanas del mundo. Es por ello, que, a través del marco SAFE se establecen y mejoran los acuerdos de la red Aduana-Aduana con el propósito de facilitar el movimiento de mercancías en el comercio internacional, intercambiar información de manera oportuna y precisa para la gestión efectiva del riesgo. Lo anterior trae como resultado la detección de envíos de alto riesgo, mejora en los controles de las cadenas logísticas y mejor eficiencia en la asignación de recursos aduaneros (OMA, 2018). Por otra parte, el marco SAFE es un instrumento integral, porque implica a los sujetos operativos desde las diferentes esferas, asegura la cadena logística y reduce el nivel de riesgo país. Toda esta integración sobre una base normativa jerárquica que los Estados asumen para que el comercio internacional se armonice.

\section{Estructura de la aduana mexicana}

En México, la ACA dirige los destinos de las cuarenta y nueve aduanas del país, a su vez, depende orgánicamente del Servicio de Administración Tributaria (SAT), que es un órgano descentralizado de la Secretaría de Hacienda y Crédito Público (SHCP). Respecto a ello, la principal función de la ACA es asegurar el cumplimiento de las disposiciones que en materia de comercio internacional se emitan. Lo anterior tiene relación con hacer cumplir las leyes aplicables y aquellas relacionadas, recaudar impuestos, aprovechamientos y derechos en materia de comercio exterior (INAP y SAT, 2012). 
Con relación a lo anterior, la seguridad del comercio internacional toma especial relevancia para la AGA, por lo tanto, a través de las directrices de prevención y control, colabora con la seguridad nacional. Además, enfatiza que, en materia de control y seguridad de comercio exterior, los proyectos y acciones se encuentran enfocados a fortalecer el control y combatir la introducción de mercancías no declaradas o prohibidas, por ejemplo, drogas, armas, explosivos, lavado de dinero, piratería y contrabando. Mediante la incorporación de sistemas tecnológicos, entre otros (INAP y SAT, 2012).

\section{Control de mercancias y mapeo de sanciones}

Por último, se ejemplifican las sanciones respecto de las mercancías antes señaladas, a través del estudio de los problemas suscitados en otros países. La presidencia Estonia del Consejo de la Unión Europea, creó el mapa de sanciones de la Unión Europea el 29 de septiembre de 2017. El mapa refleja de manera clara las sanciones y diversas situaciones aplicables al caso concreto (Comisión Europea, 2020).

Respecto a Irán, se aplican medidas restrictivas con relación a la no proliferación de armas de destrucción en masa. Estos se refieren, entre otras cosas, al embargo de armas, las medidas restrictivas relacionadas con la tecnología de misiles, las restricciones a determinadas transferencias y actividades relacionadas con la energía nuclear y las disposiciones relativas a determinados metales y software que están sujetos a un régimen de autorización.

Además, en 2006 el Líbano solicita la fuerza provisional de la ONU y despliega sus fuerzas armadas al sur del territorio. Más tarde el Consejo de Seguridad de la ONU sanciona a favor del gobierno libanés al solicitar el embargo de cualquier arma no autorizada por el gobierno en su territorio (Comisión Europea, 2020).

Como último ejemplo, se presenta el caso de Irak, donde de igual forma se aplican medidas restrictivas. El Consejo de Seguridad de la ONU adoptó un embargo financiero y comercial en 1990 , posterior a la invasión de Kuwait el 2 de agosto de 1990 por las fuerzas militares de Irak. El 22 de mayo de 2003, el Consejo de Seguridad levantó todas las medidas restrictivas contra Irak, excepto por el embargo de armas. Además, las restricciones al comercio derogadas fueron reemplazadas por otras específicas que se aplicaban al producto de todas las ventas de exportación de petróleo y gas natural del Irak y al comercio de bienes pertenecientes al patrimonio cultural de este país (Comisión Europea, 2020).

\section{CONCLUSIONES}

Los mandamientos internacionales de la OMA respecto al Marco SAFE, las resoluciones emitidas por la Asamblea General y el Consejo de Seguridad de la ONU, tanto de los regímenes de control de exportaciones, la organización y funcionalidad de la aduana mexicana, todo bajo un enfoque realista, ha sido explicado de forma general en el cuerpo del presente artículo. Por lo que se hacen las siguientes consideraciones:

En primer lugar, hay una coincidencia de los Estados que con el tiempo se ha fortalecido, el interés de estandarizar y optimizar la seguridad internacional. En segundo lugar, los Estados y los Organismos Internacionales como la OMA y la ONU, demuestran su interés a través del marco jurídico internacional, que está en una constante simbiosis. Por otra parte, la relación simbiótica de los países con objetivos bélicos e interesados en la producción, distribución, manejo, almacenamiento y transporte de mercancías consideradas como riesgo para la humanidad. En tercer lugar, el gobierno mexicano publicó el 16 de junio de 2011 en el Diario Oficial de la Federación (DOF), el acuerdo que regula la exportación de armas y tecnologías objeto de control.

Este compromiso convertido en derecho positivo vigente posibilita que la aduana mexicana esté a un nivel óptimo de actualización, cumplimiento y colaboración con la seguridad nacional de nuestro país (Secretaría de Gobernación de México, 2011). En cuarto lugar, las aduanas tienen la capacidad tecnológica para detectar operaciones que impliquen riesgo, pues se basan en información previamente analizada. Debido a la naturaleza de algunos de los bienes descritos con antelación, y que son proclives a los regímenes de control de exportación (ver Tabla 1), su detección implica un significativo grado de dificultad, porque estas no pasan por el control aduanero. En quinto lugar, para que los regímenes de control de exportaciones puedan mantenerse y ser eficaces, no basta el cúmulo de normas jurídicas, acuerdo o tratados. En ese sentido, es necesaria la voluntad de los Estados para que equilibren las políticas estableci- 
das, en pro de contribuir significativamente con la disminución de la inseguridad internacional.

En sexto lugar, falta mejorar el análisis de riesgo en las aduanas para detectar lo que no pasa por ellas. Posiblemente, una aduana cibernética con objetivos claros y alineados a combatir este tipo de delitos. Sin embargo, la falta de consenso de los países por cuestiones de economía, seguridad, comercio, entre otros factores, no permiten formular políticas uniformes para la prevención del riesgo, específicamente de las mercancías implícitas en los regímenes antes descritos.

En séptimo lugar, los esfuerzos realizados por los organismos internacionales descritos en el presente artículo enfocan sus objetivos a la seguridad internacional, donde los Estados actualizan la normativa nacional en virtud de estos cambios. Las aduanas como receptoras y emisoras de bienes se alinean al cumplimiento normativo de fiscalización.

Finalmente, la iniciativa pública se debe enfocar en elevar los estándares de seguridad a todos niveles, $y$, por otra parte, que los sectores productivos relacionados a la producción, almacenamiento, uso, distribución, transporte, entre otros, no vean impedido su desarrollo y crecimiento.

\section{REFERENCIAS}

Bernal, C. A. (2010). Metodología de la investigación administración, economía, humanidades y ciencias sociales. Recuperado de file. C:/ Users/Usuario/Downloads/Metodologia\% 20de $\%$ 20la\% 20Investigacion $\%$ 203ed ici $\%$ C3\% B3n\% 20Bernal, 20(1).

Comisión Europea (2020, 20 de diciembre). Mapa de sanciones de la Unión Europea. https:// www. sanctionsmap.eu/\#/main?checked=\&filters=\%7B\%22adopted_by.id\%22:\%7B

Díaz, T. (2014). Los regímenes de control de transferencias de tecnologías sensibles. En Instituto Español de Estudios Estratégicos (Ed.). Cuadernos de estrategia 169 Desarme y control de armamento en el siglo XXI: limitaciones al comercio y a las transferencias de tecnología (215-250). Ministerio de Defensa España. https://dialnet.unirioja.es/ejemplar/381452
Hasenclever, A.; Mayer, P. y Rittberger, V. (2000). Integrating Theories of International Regimes. Review of International Studies, 26 (1), $3-33$.

Hernández, R., Fernández, C., y Baptista, P. (2014). Metodología de la investigación. McGraw Hill.

Hurtado, J. (2010). Guía para la comprensión holística de la ciencia. Fundación Sypal.

Instituto Nacional de Administración Pública, A. C. - INAP y Servicio de Administración Tributaria (2012). Consultoría para la "Evaluación de procesos de los programas presupuestarios del SAT 2012. http://omawww.sat.gob.mx/ fichas_tematicas/Documents/Control_operacion_aduanera2012.pdf

Krasner, S. (1982). Structural causes and regime consequences: regime as intervening variables. International Organization, 36 (2), 185205.

Lascurain, F. (2018). Teoría de regímenes internacionales: el caso del recurso agua en el régimen ambiental México-Estados Unidos. Si Somos Americanos. Revista de Estudios Transfronterizos, 18(2), 128-158.

Lechuga-Cardozo, J. I.; Leyva-Cordero, O., y Núñez-García, A. (2019). Internationalization strategy in the handicraft industry: the Galapa-Colombia case. Revista Academia a Negocios, 5(2), 99-106.

Lechuga-Cardozo, J. I., Núñez-García, A., y Leyva-Cordero, O. (2019). Contexto Sociojurídico de la Lex mercatoria en Colombia. Revista Ciencia Jurídica y Política, (6), 42-56.

Mesinas, M. (2017). Regímenes Internacionales, una herramienta teórica para comprender la implementación de los derechos culturales en México, ante la creación de la Ley general de Cultura. Cuicuilco. Revista de Ciencias Antropológicas, 24 (68), 231-256.

Naciones Unidas (2020, a). Capítulo V. El Consejo de Seguridad. https://www.un.org/es/sections/un-charter/chapter-v/index.html.

Naciones Unidas (2020, b). Consejo de Seguridad de las Naciones Unidas: Funciones y poderes. https://www.un.org/securitycouncil/content/functions-and-powers

Naciones Unidas (2020, c). Información general. Naciones Unidas. https://www.un.org/es/ sections/about-un/overview/index.html 
Naciones Unidas (2020, d). Órganos principales. https://www.un.org/es/sections/about-un/ main-organs/index.html

Naciones Unidas Wiki (2020, e). Consejo de Seguridad: Historia. https://nacionesunidas. fandom.com/es/wiki/Consejo_de_Seguridad

Naciones Unidas (2007). Consejo de Seguridad 2006: Recapitulación. https://www.un.org/ es/documents/sc/scaction/2006/

Organización Mundial de Aduanas - OMA. (2018). Marco de Norma SAFE (Ed. 2018). http:// www.wcoomd.org/en/topics/facilitation/ instrument-and-tools/tools/ /media/C6CDF626AFB348FCA2AC59B796B79833.ashx

Person S., F. y Rochester., J. M. (2000). Relaciones Internacionales. Situación Global en el Siglo XXI. Ed. McGraw Hill.

Puchala, D. J. y Hopkins, R. F. (1983). International Regimes: Lessons From Inductive Analysis. En S. D. Krasner (Ed.). International Regimes (pp. 61-91). Oxford University Press. https:// works.swarthmore.edu/fac-poli-sci/241

Schiavon, J., Ortega, A., Lopez-valejo, M. y Velazquez, R. (2014). Teorías de las Relaciones Internacionales en el siglo XXI: Interpretaciones críticas desde México. Primera edición. BUAP, COLSAN, UABC, UANL \& UPAEP.
Secretaría de Economía (2021). Regímenes internacionales. http://www.siicex.gob.mx/portalSiicex/CONTROL\%20DE\%20EXPORTACIONES/inicio.html

Secretaría de Gobernación de México (2011). Acuerdo por el que se sujeta al requisito de permiso previo por parte de la Secretaría de Economía la exportación de armas convencionales, sus partes y componentes, bienes de uso dual, software y tecnologías susceptibles de desvío para la fabricación y proliferación de armas convencionales y de destrucción masiva. Diario Oficial de la Federación - DOF 16 de junio del 2011. https://www.dof.gob. $\mathrm{mx} /$ nota_detalle.php?codigo $=51962268 \mathrm{fe}-$ cha $=16 / 06 / 2011$

Velázquez, R. y González, S. (2016). Realismo clásico. En J. Schiavon; A. Ortega, M. López-Vallejo y R. Velázquez. Teorías de las relaciones internacionales en el siglo XXI. (pp. 251-263). Editorial CIDE.

Zeraoui, Z., Sarquis, D., Gonzalez, L., Perni, O., Telman, P. y Balderrama, R. (2014). Los procesos Mundiales Contemporáneos: Un Mundo Transformado. Trillas. 
